\title{
Forum ZV
}

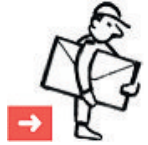

\section{Zum Editorial zu eHealth [1]}

Sehr geehrte Frau Gauthey

Was mir nicht ganz klar ist: Wieso sind die Verlautbarungen von SGAM.informatics und FMH eHealth zum Teil so widersprüchlich? Wenn es wichtig ist, dass eHealth-Standards von Ärzten mitentwickelt werden, wieso gibt es dann keine ersichtliche Absprache zwischen eHealth-Strategen und eHealth-Praktikern in der Schweiz? Gut, vielleicht gibt es ja hinter den Kulissen eine Zusammenarbeit, von der ich bisher nichts wahrgenommen habe. Dr. Bhend wird da mehr darüber wissen.

Was ich aber sicher weiss: Die auf meinen Leserbrief [2] von 2007 (von Ihrem Vorgänger Dr. Giger) versprochene öffentliche Publikation des API [3] und eines SDK [4] gab es nicht. Auf mehrere entsprechende E-Mail-Anfragen bekam ich zumindest keine Antwort. Inzwischen ist die HPC einfach ein für teures Geld nochmals erfundenes Rad, das ohne echten Mehrnutzen neben der SuisseID herrollt und ASAS [5] einzuholen versucht. So richtig verstehen kann ich die 12000 Kollegen nicht, die nun stolze Besitzer einer HPC sind. Würde mir bitte jemand von Ihnen mal schreiben, was genau Sie nun mit dieser Karte machen, damit ich mir ein besseres Bild vom Nutzen machen kann?

Gerry Weirich, Innere Medizin FMH, Schaffhausen

1 Gauthey M. eHealth: die Rechte der Patienten und der Ärzteschaft schützen.

Schweiz Ärztezeitung. 2010; 91(21):721.

2 Weirich G, Giger M. Health Professional Card der Schweizer Ärzteschaft ante portas. Schweiz Ärztezeitung. 2007;88 (50): 2124-5).

$3 \mathrm{API}=$ Application Programming Interface $=$ Programmierschnittstelle.

4 SDK = Software Development Kit. «Werkzeugkasten» für die Software-Entwicklung, in diesem Fall ein spezieller Werkzeugkasten für die FMH-HPC.

5 ASAS = «Arpage Security and Access Services» Bezeichnung einer Software, welche für den sicheren Datenaustausch im Gesundheitswesen im Rahmen des Health-Info-Net (HIN) eingesetzt wird.

\section{Antwort}

Sehr geehrter Herr Weirich

Ihre Fragen zeigen deutlich auf, welche Unsicherheiten bei der Ärzteschaft im Zusammenhang mit der HPC der FMH (Health Professional Card) bestehen. Gerne gehe ich etwas näher darauf ein: Diese FMH-HPC dient den Ärztinnen und Ärzten vor allem dazu, sich als Fachperson auszuweisen: zunächst visuell, aber darüber hinaus auch für alle Leistungen im Bereich eHealth. Die FMH hatte sich entschieden, die Karte zu entwickeln, bevor alle Optionen klar waren, denn sie wollte an vorderster Front mitbestimmen! Momentan wirkt die Karte deshalb vielleicht noch wie eine Gold Card, deren Preis angesichts des Nutzens, den sie aktuell bietet, zu hoch erscheint. Doch wir stehen knapp davor, sie auf breiterer Basis einsetzen zu können. Im Rahmen der Umsetzung der nationalen Strategie eHealth stützen sich Modellversuche für die Authentifizierung der Ärztinnen und Ärzte auf die FMH-HPC. Mit dieser Karte setzt die Ärzteschaft ein klares Zeichen für eine abgesicherte Kommunikation über das Internet, und HIN ist daran, sie in seine Plattform zu integrieren. Es handelt sich um eine Karte mit offenen Funktionen, die sich somit noch weiterentwickelt lässt (zum Beispiel durch das Aufladen von qualifizierten Zertifikaten oder des/der Card Verifiable Certificate(s) CVC oder durch die Entwicklung von Karten für das Personal oder andere Berufsgruppen). Sie ergänzt die Karte von SuisseID, die ebenfalls ein qualifiziertes Zertifikat anbietet. Das Card API ist auf myFMH verfügbar, und die Spezifikationen der HPC wurden bereits letztes Jahr auf der Website der FMH veröffentlicht.

So weit zu den praktischen Aspekten. Sie werfen jedoch auch die Frage auf, welche Verbindungen zwischen der Karte und der Strategie der FMH im Bereich eHealth bestehen: Die Verwendung eines sicheren Identifikationsmittels bildet Teil der nationalen Strategie eHealth, und die Ärztinnen und Ärzte wollten, dass diese Identifikation nicht über individuelle Lösungen, sondern über eine einheitliche Lösung für die gesamte Ärzteschaft entwickelt wird. Auf dieser Grundhaltung beruht das Projekt HPC der FMH. SGAM.Informatics («Hausärzte Schweiz») hat sich an den Diskussionen beteiligt, die im Zusammenhang mit dem Projekt geführt wurden. Sie sind in der
Arbeitsgruppe eHealth der FMH vertreten, die die strategischen Ziele der FMH in diesem Bereich erarbeitet hat und die erforderlichen Stellungnahmen $\mathrm{zu}$ verschiedenen Themen formulieren wird, unter anderem im Rahmen der Vernehmlassungen und Anhörungen auf Bundesebene. SGAM.Informatics engagiert sich vorzugsweise in einem praktischen und sich mit der Karte ergänzenden Bereich, den Programmen für die Arztpraxis, zum Beispiel elektronischen Patientendossiers.

Sie setzen sich dafür ein, dass bestimmte Standards eingehalten werden, die für die Ärzteschaft unerlässlich sind. Dafür sind wir ihnen zu Dank verpflichtet.

Monique Gauthey, Fachärztin FMH, Mitglied des Zentralvorstands der FMH, Verantwortliche für das Ressort eHealth 